\title{
To Assess the Effectiveness of Progressive Muscle Relaxation Technique in Reducing Stress Among Primary Infertile Women Undergoing Intra Uterine Insemination
}

\author{
Kavita J. Gomase ${ }^{1}$, Manjusha Mahakarkar ${ }^{2}$ and Pooja Kasturkar ${ }^{3}$ \\ ${ }^{1,2}$ Department of Obstetrics and Gynaecology Nursing, SRMM College of Nursing, Datta Meghe Institute of \\ Medical Sciences, (Deemed to be University), Sawangi Meghe, Wardha, Maharashtra, India \\ ${ }^{3}$ Department of Mental Health Nursing, SRMM College of Nursing, Datta Meghe Institute of Medical Sciences, \\ (Deemed to be University), Sawangi Meghe, Wardha, Maharashtra, India
}

\begin{abstract}
Rachel's eloquent plea ringing through centuries conveys clearly, the desperate hope of the infertile women and their need. The term infertility refers to opponent inability of a couple to conceive after one year of unprotected intercourse. 60-80 million people experience infertility around the world and most of those people live in developing countries. In India infertility affects 10-15\% of couples in reproductive age group. Infertility is called "primary" when there has been no prior conception. Male is responsible for the infertility in about 65\%. Female is responsible for the infertility in about $40-50 \%$ of the cases. Incidences of infertility in India are 92\% primary and 8\% secondary. To assess the effectiveness of progressive muscle relaxation therapy in reducing stress before and after intrauterine insemination in both experimental and control group. True - experimental research design, Pre-test - post-test -only design was done from July 2020 November 2020 in 49 primary infertile women undergoing intra uterine insemination. Primary infertile women were randomly assigned to either the Control group or the intervention group. Women were observed for a total duration of four months and assessed with the perceived stress scale. Analysing the data was done using SPSS software, version 16, and paired t, independent t, chi-square and Mann-Whitney tests. Total 49 women were analysed in two groups i.e. intervention group $(n=24)$ and control group $(n=25)$. There were no significant differences in age, education level, spouse's education level, occupation status, spouse's occupation status, cause of infertility, marriage duration, the length of time one knows about their infertility, and the duration of treatment between two groups. After the intervention (Progressive muscle relaxation therapy) at 9th day, 15th day of menstruation and after intra uterine insemination, the level of stress has shown a statistically significant reduction $(\mathrm{p}=0.000)$. This interventions were more effective for reducing the level of stress. The findings showed that Progressive Muscle Relaxation technique can be benefited to reduce the level of stress among primary infertile women who are undergoing Intra Uterine Insemination
\end{abstract}

\section{KEY WORDS: PROGRESSIVE MUSCLE RELAXATION, PRIMARY INFERTILE WOMEN.}

\section{INTRODUCTION}

Infertility represents complex physical, spiritual, emotional crises and affects all areas of the women's health and wellbeing, the co-existence of infertility and psychological distress is supported by sound scientific evidence. However the casual direction may well be

Biosc Biotech Res Comm P-ISSN: 0974-6455 E-ISSN: 2321-4007

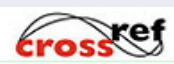

Identifiers and Pagination

Year: 2021 Vol: 14 No (9) Special Issue

Pages:66-69

This is an open access article under Creative

Commons License Attribn 4.0 Intl (CC-BY).

DOI: $h t t p: / / d x$.doi.org/10.21786/bbrc/14.9.15 from infertility to psychological stress. The degree of psychological stress can be very high and it is essential that, this to be recognized. Infertility myths still exist today. Infertility is often viewed as a failure of the women and to a lesser extends the man, to fulfill their role in society. Studies suggest that infertile women typically do not seek medical help until after more than a year of trying to conceive unsuccessfully. The process of seeking infertility treatment has increased in recent years because of the development of new techniques for treating infertility. This innovations and advancements pose challenges for the nursing personnel while caring the infertile women. So she must understand the dynamics of infertility, different treatment modalities and help 
the infertile women to cope with their psychological distress.

Infertility is often equated with pain and stress in our society. In India a women without child is considered as "Barren". It is often experienced as the most stressful event in their lives. In infertility problems have historically been attributed to women. Given the plethora of available infertility treatment and their complexity, infertile women and physicians may become so engaged in physiological concerns that they fail to attend to psychological aspects of the process. They focus their interest on the technology to the exclusion of all other concern. These medicalizations of the crisis of infertility fail to adequately address psychological aspect of the women's experience.

A review of literature shows that, health personnel should assess the level and source of existing stress and determine the appropriate intervention to reduce the psychological stress. In the area of infertility nurses are faced with challenges, to provide comprehensive health care in a cost effective manner. Relaxation is one of the cost effective simple and non-invasive strategies to relieve stress.The relaxation training is also within the scope of nursing practice. It does not need physician's supervision and can be practiced in variety of settings. The need for the study arises from the fact that 40-50 infertile women come for intra uterine insemination at Srusthi Medical foundation as per the 2004 statistics. According to the present practice in this hospital there is no specific intervention for stress reduction in infertile women undergoing treatment7. The Betty Neumann System model 2002 also classified the nurse's role to reduce the stressors reaction through use of primary, secondary or tertiary prevention as interventions to retain, attain and maintain an optimum wellness level. This study proposes to determine whether progressive muscle relaxation technique is effective in reducing the level of stress and if so, whether, it can be incorporated as a regular nursing intervention in the care of primary infertile women undergoing intra uterine insemination.

Aim Of The Study: The aim of the study to assess the effectiveness of progressive muscle relaxation therapy in reducing stress before and after intrauterine insemination in both experimental and control group.

H1: There is an individual difference in level of stress experienced by primary infertile women undergoing intra uterine insemination with post test score.

\section{MATERIAL AND METHODS}

Study design, participants and setting: This was True -experimental research design, Pre-test -post-test -only design with prospective participants recruited from Infertility and artificial insemination clinic at selected hospitals in Wardha district being assigned to intervention and control group. Primary infertile women were eligible to participate of the age group of
25 - 35 years, Primary infertile women who can read and write Marathi and Hindi, Women with the diagnosis of primary infertility and undergoing intra uterine insemination with the partner's sperm and combined with ovulation induction, Primary infertility with or without altered semen analysis in the partner. Women were excluded if Women with pre-existing illnesses including hypertension, cardiovascular diseases, diabetic mellitus, thyroid disease and renal disease, Women with secondary infertility.

Randomisation: All primary infertile women who were undergoing intra uterine insemination allocated randomly into control and experimental group by block randomization. Every ten consecutive patients from a block and randomization was done in such a way that within each ten patients ( block ) five patients were randomly allotted to experimental group and five to the control group. This type of procedure is called block randomization. Among next 10 patients 5 were randomly allocated to experimental and the other 5 to the control.

Intervention and procedures: One of the data collection tools in this study was demographic questionnaire, including personal and family information on age, education, spouse's education, occupation, spouse's occupation, and marriage duration. Another tools for data collection was the Perceived Stress Scale. The Perceived Stress Scale (PSS) is the most widely used psychological instrument for measuring the perception of stress. It is a measure of the degree to which situations in one's life are appraised as stressful. Items were designed to tap how unpredictable, uncontrollable, and overloaded respondents find their lives. The scale also includes a number of direct queries about current levels of experienced stress. PSS scores are obtained by reversing responses (e.g., $0=4,1=3,2=2,3=$ 1 \& $4=0$ ) to the four positively stated items (items 4 , 5,7 , \& 8 ) and then summing across all scale items. A short 4 item scale can be made from questions 2, 4, 5 and 10 of the PSS 10 item scale. In this study, level of stress was assessed by Perceived stress scale to the both groups, before the first session as a pre-test. Then, it was completed 9th day to 14th day of menstrual cycle as a post-test. In the intervention group, progressive muscle relaxation therapy was introduced in six sessions and in the control group, only routine care was given.

In intervention group, the study participants, were lying on the side with their legs fairly bent (no to put pressure at any of the muscles) and in a relax position with closed eyes. In Jacobson technique, the body muscles were divided into eight parts and during performing the technique, an active muscle contraction for 5 seconds and then release of the same muscles for 30 seconds was implemented. The order of muscle contraction and release were as following: right foot, left food, right hand, left hand, stomach and back, chest and the shoulders, face, head and scalp. The first relaxation technique in the intervention group was performed around the 9th day of menstrual cycle and then every other day until women 
were undergone Intra uterine insemination (totally 6 sessions) in the presence of the researcher in Infertility and artificial insemination clinic at selected hospitals in Wardha district. After 15 days, post test was conducted with Perceived stress scale.

Ethical Consideration: The approval for the study was taken from the Institutional review board and the Ethical Committee of DMIMS (Deemed to be University). Informed consent was taken from all the patients before they underwent the procedure.

Statistical Analysis: The SPSS software (Statistical Package for the Social Sciences, Version 21.0, SPSS were used to analyse the data. To assess the homogeneity of the two groups with respect to the demographic and confounding variables, Chi-squared test, and independent t-test were used. To compare the stress mean score of the two groups, independent t-test was used. p-value $\leq 0.05$ was considered as the significant level.

\section{RESULTS}

Section I: There were total 49 primary infertile women participated in this study in two group At last 49 women were analysed in two groups i.e. intervention group $(n=24)$ and control group $(n=25)$. There were no significant differences in age, education level, spouse's education level, occupation status, spouse's occupation status, cause of infertility, marriage duration, the length of time one knows about their infertility, and the duration of treatment between two groups (table no.1).

\begin{tabular}{|l|c|c|c|}
\hline \multicolumn{4}{|c|}{ Table 1. Distribution of characteristics of the study population } \\
$\begin{array}{l}\text { Demographic } \\
\text { variables }\end{array}$ & $\begin{array}{c}\text { Interventional } \\
\text { group }\end{array}$ & $\begin{array}{c}\text { Control } \\
\text { group }\end{array}$ & p-value \\
\hline Age & & & \\
\hline 20-30 & $9(37.4)$ & $11(44)$ & 0.609 \\
$30-40$ & $14(58.3)$ & $13(52)$ & \\
40 above & $1(4.2)$ & $1(4)$ & \\
\hline Level of education & & & \\
\hline Primary education & $5(20)$ & $2(8)$ & 0.439 \\
Secondary education & $9(38.3)$ & $11(44)$ & \\
Graduation & $10(41.7)$ & $12(48)$ & \\
\hline Spouse's education level & & & \\
\hline Primary education & $2(8.4)$ & $3(12)$ & 0.751 \\
Secondary education & $7(29.2)$ & $9(36)$ & \\
Graduation & $15(62.4)$ & $13(52)$ & \\
\hline Occupation status & & & \\
\hline Housewife & $19(79.2)$ & $17(68)$ & 0.427 \\
Working & $5(20.8)$ & $8(32)$ & \\
\hline Spouse's occupation status & & & \\
\hline Employee & $15(62.5)$ & $12(48)$ & 0.371 \\
Business & $6(25)$ & $11(44)$ & \\
Farmer & $3(12.5)$ & $2(4)$ & \\
\hline Causes of infertility & & & \\
\hline Male factor & $10(41.7)$ & $11(44)$ & 0.263 \\
Female factor & $5(20.8)$ & $2(8)$ & \\
Both & $6(25)$ & $4(16)$ & \\
Unknown & $3(12.5)$ & $8(32)$ & \\
\hline
\end{tabular}

Section II: The result shows that level of stress had no significant difference between two groups before intrauterine insemination $(p=0.749)$. But after the intervention (Progressive muscle relaxation therapy) at 9th day, 15th day of menstruation and after intra uterine insemination, the level of stress has shown a statistically significant reduction $(p=0.000)$. This interventions were more effective for reducing the level of stress. (Table 2)

\section{DISCUSSION}

A study was conducted to determine the efficacy of progressive muscle relaxation on reducing stress of the infertile women undergoing treatment. Methods: 64 infertile women were taken as sample, in that 32 were taken for experimental, 32 were taken for control. Results: there was a significant reduction in systolic blood pressure was observed, a significant reduction on 3 or 5 State-Trait Anger Expression Inventory Scales. Conclusion: Progressive muscle relaxation technique appears to be an effective method to reduce anger, 
maintain blood pressure and promote psychological well being.

A study done on testing the feasibility and effectiveness of incorporating progressive muscle relaxation technique into routine instruction on infertile women undergoing treatment. Design: A quasi-experimental design Subjects: 33 women were taken for study Results: 40\% of women reported that they had a good relaxation during procedure, they were relieved from stress and felt happy. Conclusion: Based on above result,it is understood that progressive muscle relaxation technique reduce the anger and worries and promotes psychological wellbeing.

Table 2. Effectiveness of progressive muscle relaxation therapy in reducing stress before and after intrauterine insemination in both experimental and control group.

\begin{tabular}{|l|l|c|l|l|}
\hline \multirow{2}{*}{ Variable } & & Intervention group & Control group & p-value \\
\hline \multirow{2}{*}{ Level of stress } & Before intra uterine insemination & $166.75 \pm 13.27$ & $167.92 \pm 12.14$ & 0.749 \\
\cline { 2 - 5 } & 9th day of menstrual cycle & $115.75 \pm 13.88$ & $177.12 \pm 19.37$ & 0.000 \\
\cline { 2 - 5 } & 15th day menstrual cycle & $118.08 \pm 15.37$ & $179.40 \pm 18.34$ & 0.000 \\
\cline { 2 - 5 } & After intra uterine insemination & $120.50 \pm 16.24$ & $183.76 \pm 14.97$ & 0.000 \\
\hline
\end{tabular}

A study was conducted on infertility related to stress in men and women predicts the treatment outcome one year later. Design: Cohort study Subjects: 818 samples were taken. After one year the outcome was poor in women with lot of marital problem. Results: A study result shown that women with more marital distress require more treatment to conceive than women with less marital distress $77 \%$ of women with less marital problem showed good results.

A study done on perceived infertility related stress and its relationships to the marital adjustment and depression in infertile couples to explore the relationships between partner and infertility related stress and effect on marital adjustment in infertile men and women. Subject: 50 sample were taken for study, 25 sample were experimental,25 sample were control group. Results: 15 mothers reported that they had a good marital satisfaction among experimental group.Conclusion: They reported that it helped them to relieve from stress and successfully manage the stressful event. Above studies support to the present study.

\section{CONCLUSION}

The findings showed that Progressive Muscle Relaxation technique can be benefited to reduce the level of stress among primary infertile women who are undergoing Intra Uterine Insemination

Conflict of Interest: Nil

Source of Funding: Self
Ethical Clearance: The study was approved by the Ethics committee of the institute.

\section{REFERENCES}

Alexander. (1996) Effects of relaxation in reducing dyspnoea and anxiety among COPD and asthma. Indian nursing council of psychological medicine.

Christopher.(1999) Psychological assessment and follow up after IVF - assessing the impact of failure, fertility and sterility.

Eller.(1998) Perceptions of infertility and treatment stress in females as compared with males entering IVF treatment. The journal of fertility and sterility.

Ellne,(2003) Study on infertility related to stress in men and women".

Fulton, Kathryn Blauvelt "The effects of music therapy on physiological measures,perceived fatigue and stress on infertile mother."

Gjuzette. A review of relaxation technique. Buller worth publisher.

Henry,(1998) "Study on perceived infertility related stress in infertile couples."

Hillary-Klonoff-Cohen. (1998) Obstetrics and gynecology. Philadelphia. St Louis.

Simbach/Inn,"Study to determine the efficacy of progressive muscle relaxation on infertile mother".

Smith BW “ Effectiveness of incorporating progressive muscle relaxation technique on infertile women undergoing treatment"

Smith BW, Shelly BM, Dalen J Wiggins K “Department of psychology” University of New Mexico.

Tittle baun. (1988) Relaxation therapy - cancer nursing. Philadelphia. Lippincott.. 